- Starke internationale Orientierung: der Großteil der Vorschläge beruht auf einer Anpassung erprobter ausländischer Modelle an die österreichische Situation

- In der Zusammensetzung der KI: Balance von Gemeinsamkeit (kriminalpolitisches Grundverständnis) und Verschiedenheit in den professionellen Zugängen (Recht, Sozialwissenschaft, Sozialarbeit)

- Das Zusammenspiel der verschiedenen Kapitalien der KI-Mitglieder (theoretisches und praktisches Wissen, Feldkompetenz, Reputation, Beziehungen zu Multiplikatoren und Entscheidungsträgern) ergibt eine potente Gruppe.
- Zivilcourage: Wenngleich kein KI-Mitglied ein höheres berufliches Risiko einging, war einigen ein „Naserümpfen“ oder „hochgezogene Augenbrauen“ von KollegInnen und Vorgesetzten sicher.

- Kontinuität auf Sparflamme: Es wurde ein ökonomischer und arbeitsteiliger Arbeitsmodus gefunden, der die einzelnen Mitglieder relativ wenig belastete.

- Kommunikation der Vorschläge auf verschiedenen Kanälen: Pflege vorhandener Kontakte zu JournalistInnen in Verbindung mit drei Pressekonferenzen und einer Presseaussendung, informelle Kontakte zu Justizpolitikern und Beamten des Justizministeriums, Vorsprachen bei drei verschiedenen MinisterIn- nen, Referate auf Tagungen und Publikationen in Fachzeitschriften.

- Parteipolitische Äquidistanz: Alle Vorschlägen beruhen auf kriminalpolitischen Überlegungen, die zwar eine größere oder weniger große Nähe zu den Vorstellungen einzelner politischer Parteien haben, nicht aber auf parteipolitischen Positionen.

Trotz aller Bemühungen sind Initiativen wie die KI von der politischen Großwetterlage abhängig. Es kann sich aber lohnen, bei ungünstigen klimatischen Bedingungen gegensteuernd aufzutreten. Bei politischen Veränderungen sind dann rasche Teilerfolge möglich.

\title{
Hamburger Strafvollzug - Wege und Irrwege Zentrale Fakten einer verfehlten Strafvollzugspolitik
}

\section{Fünf Hauptsünden der hamburgischen Strafvollzugspolitik seit 2001}

1. Geld verschleudert: Ausbau der

JVA Billwerder um weitere ca. 400, auf rund 800 Plätze

Mit Bürgerschaftsdrucksache vom 7.5. 2002 (Drs. 17/802) beantragten Kusch/ Lüdemann 30.260.000 € für die Erweiterung der JVA Billwerder um 400 Plätze des geschlossenen Vollzuges (Billwerder II). Dieses Geld wurde in den Sand gesetzt. Zusätzlich wird Hamburg mit jährlichen Betriebsmittelkosten von ca. 1.400.000€ belastet. Wie konnte es dazu kommen? Drei Antworten:

Falsche Prognose: Kusch/Lüdemann erwarteten von 2002 bis 2004 einen Anstieg der Zahl der Gefangenen um $17 \%$ auf 3.600. Daran hielten er, seine Behörde, der Senat und die Fraktionen der Koalition fest, obwohl ihnen und der Öffentlichkeit alsbald und wiederholt der Unsinn (und die Geschichtsblindheit!) dieser Prognose vorgehalten worden war. Schon im Jahr der Mitteleinwerbung (2002) wurden nicht die vorausgesagten 3.266 (Drs. 17/802, S. 3), sondern nur 3.085 Gefangene gezählt. Bereits im Januar 2004 sank sie unter die Grenze von 3.000. Nach kontinuierlichem Rückgang seither lag sie im Durchschnitt des Jahres 2006 bei $2.549,2007$ bei 2.227 Gefangenen.

Überflüssige Haftplätze: Für diese ca. 2.250 Gefangenen sind, nach Abschluss laufender Umbauten, 3.202 Haftplätze vorhanden. „Jede vierte Gefängniszelle“, so „Die Welt“ am 6. 12. 2006, „steht leer". Es war unsinnig, Billwerder II zu bauen - insbesondere, wenn die mehr als 100 Haftplätze im Übergangsvollzug und in der Sozialtherapie erhalten geblieben wären (siehe dazu Punkt 5).

Blockierte Kontrollen: Der Vorgang rührt an Grundfragen eines sachgerechten Verwaltungshandelns. Die erste Rate für Billwerder II in Höhe von 8,8 Mio € war 2004 fällig. Genug Zeit, sollte man meinen, um die Planung anhand der Fakten zu überprüfen, zumal die Bauaufträge erst am 30. 1. 2004 erteilt wurden (s. Broschüre der Justizbehörde „So viel ist sicher" vom Februar 2006, S. 22). Aber alle Warnungen wurden missachtet. Wichtige Hamburger Medien versagten; Senat und Bürgerschaftsmehrheit winkten das Projekt durch. Die sonst äußerst strengen und fachlich versierten Kontrollen in der Finanzbehörde und im Planungsstab der Senatskanzlei blieben wirkungslos. Zu fragen ist, ob und ggf. von wem die fachbehördlichen und behördenübergreifenden Sicherungen außer Kraft gesetzt worden sind.

Was sagt die Justizbehörde heute? In einem Interview mit „Die Welt“ vom 5. 1. 2007 beklagt Senator Lüdemann die „Geschichtsvergessenheit der Opposition“. Auch Rot-Grün habe 350 Haftplätze neu errichten wollen. Jedoch gibt es zwischen den Planungen der Jahre 1999/2000 und denen von Kusch/Lüdemann der Jahre 2001/2002 drei große Unterschiede: Die Belegungsfähigkeit des geschlossenen Vollzuges sollte nach damaligen Plänen nur um 175 Plätze angehoben, weitere 175 sollten dagegen zum Abbau von Saalbelegungen genutzt werden. Angenommen wurde ein Zuwachs der Gefangenenzahlen von 2000 bis 2004 von jährlich nur einem Prozent. Selbst dies wurde, wie es Brauch war, von der Finanzbehörde und dem Planungsstab energisch hinterfragt. Das führte dazu, dass der Bau nicht vor 2004 beginnen sollte, und dass die Realisierung zwingend an den jährlichen Nachweis der Belegungszahlen gebunden wurde. Diese Sperre gegenüber fachbehördlichen Durchmarschund Geldverschwendungsgelüsten hätte das Projekt vermutlich gestoppt.

2. Umbau um jeden Preis: die offene Anstalt Billwerder wird geschlossener Vollzug

Kusch/Lüdemann haben alsbald nach Amtsübernahme angeordnet, die vom Vorgängersenat als offene Anstalt mit 380 Plätzen konzipierte Einrichtung Billwerder I in geschlossenen Vollzug umzuwandeln. Dafür wurden 14.000.000€ veranschlagt, bewilligt und vergeudet. Die Umrüstung war baufachlich überflüssig, weil auch 
für den offenen Vollzug u.a. Gitter vor den Fenstern und stabile Zäune als Umwehrung vorgesehen waren. Für dort unterzubringende, überwiegend kurzstrafige Gefangene mit eher geringer Delinquenz wäre das ausreichend gewesen.

Der nachträgliche Umbau war überschießend teuer, weil geschlossener Vollzug an kurzen Wegen orientiert kompakter und mit qualitativ besonders hochwertigen Materialien errichtet worden wäre. Das betrifft vor allem die nun überdehnte Länge der Mauern und den Aufwand für sonstige Sicherungssysteme. So belastet der nachträgliche Umbau den Haushalt mit dauerhaft erheblich höheren Personalund Wartungskosten, ohne die erhoffte, aber eben in diesem Maß auch überflüssige, Sicherheit tatsächlich zu erbringen. Die Umrüstung ist auch konzeptionell verfehlt, weil sie den offenen Vollzug über jedes sinnvolle Maß hinaus reduziert. Das sei genauer betrachtet:

\section{Die Lust am Einsperren: Der offene Vollzug als Nadelöhr}

Die Haftplätze des offenen Vollzuges wurden nach 2001 radikal reduziert. Davon sind vor allem die zu Freiheitsstrafe und Sicherungsverwahrung verurteilten Männer betroffen: Hier sank die Zahl dieser Plätze bis 2006 von $639=23 \%$ auf $178=$ 6,5\% (Stand: Juli 2006; Quelle: Antwort des Senats vom 22. 8. 2006 auf eine Kleine Anfrage, Drs.18/4826). Diese 178 Plätze befinden sich in der JVA Glasmoor und dort überwiegend in Sälen bis zu acht Plätzen. Inzwischen wurde die Zahl der Plätze des offenen Vollzuges für Männer zu Lasten einer Abteilung für straffällige Frauen auf 207 erhöht. Diese waren im Dezember 2007 mit durchschnittlich nur 147 Gefangenen belegt.

Demgegenüber waren bundesweit am 31. 3. $2006 \mathbf{1 5 , 9 \%}$ aller männlichen Strafgefangenen im offenen Vollzug untergebracht. Einige Länder liegen deutlich über dieser Zahl - vor allem Berlin (29,2\%), NRW (27,1\%) und Niedersachsen $(19,1 \%)$. Hamburg gehört nun zu den Schlusslichtern.

Diese Entwicklung ist wirtschaftlich nachteilig, wie am Beispiel Billwerder I gezeigt wurde. Sie ist zudem fachlich falsch, weil der potentiell schädliche, hoch gesicherte geschlossene Vollzug für viele Gefangene nicht erforderlich ist. So verbüßen gut $40 \%$ der Strafgefangenen Strafen lediglich bis zu maximal einem Jahr, rund $65 \%$ bis höchstens zwei Jahre. Aber gerade auch Gefangene mit langen Strafen müssen i.d.R. gegen Ende der Verbüßung über den offenen Vollzug auf die Freiheit vorbereitet werden. Eine baulich gut gerüstete und sinnvoll umwehrte offene Anstalt bietet für diese Aufgaben ein angemessenes Sicherheitsniveau. Dies um so mehr, je stringenter Gefangene in ein intensives Beschäftigungs- und Eingliederungsangebot eingebunden werden. Schließlich wird durch den Vorrang der Verwahrung der spezialpräventive Auftrag des Vollzuges verfehlt und die Gefahr des Rückfalls erhöht. So wird nicht nur menschliches Leid gemehrt, es entstehen auch hohe Kosten weit über die des justizförmigen Verfahrens hinaus. Das zeigt sich noch deutlicher, wenn die Entwicklung der Vollzugslockerungen insgesamt hinzugenommen wird:

\section{Abbau von Eingliederungschancen: Ausgang, Urlaub, Freigang als Aus- nahme}

Warum Vollzugslockerungen? Ausgang, Urlaub und Freigang im Sinne des Strafvollzugsgesetzes (StVollzG) von 1977 sollen dazu beitragen, schädliche Folgen der Einsperrung durch lebensnahe Gestaltung des Vollzuges zu verringern und förderungswürdige Bezüge zur Familie und anderen Bezugspersonen aufrecht zu erhalten und/oder neu zu knüpfen. Sie ermöglichen es, Gefangene zur Entlassung hin in zunehmenden Freiräumen zu erproben und bei der Gestaltung eines sozialen Empfangsraums nach der Entlassung (Arbeit, Wohnraum, Schuldenregulierung etc.) zu unterstützen. Diese Aufgabe ist aufwändig und für Bedienste und Gefangene i.d.R. anstrengender, als die bloße Verwahrung bis zum Tage der Entlassung. Die Gefahr des Missbrauchs ist sorgfältig zu ermitteln. Jedoch darf das nicht dazu führen, schwierigere Fälle pauschal unvorbereitet und unerprobt zu entlassen und mit dem dadurch vergrößerten Rückfallrisiko allein die Öffentlichkeit zu belasten. Im übrigen haben wissenschaftliche Untersuchungen belegt, dass auch bei einem intensiven Einsatz von Lockerungen schwere Straftaten die absolute Ausnahme sind und sich im Promillebereich bewegen. Dem Missbrauchsrisiko wäre der deutlich resozialisierende Effekt insbesondere eines mit Freigang, d.h. mit Arbeit oder Ausbildung aus der Anstalt heraus abschließenden Eingliederungsvorgangs gegenüber zu stellen.

Entwicklung in Hamburg: 2006 wurde der tiefste Punkt erreicht: Im Vergleich mit 2001 gingen die Gewährungen von Ausgang um 59,3\%, von Urlaub um $68,8 \%$ und des Freigangs um 64,5 \% zurück. Die absolute Zahl der Teilnehmer am Freigang sank von 484 auf 172 und damit die Zahl derjenigen, die als sonst schwer Vermittelbare mit Arbeit entlassen worden sind und Haftkosten, Sozialversicherungsbeiträge und ggf. Familienunterhalt und Schulden bezahlt haben. Im Jahr 2007 zeichnete sich eine Trendwende auf niedrigem Niveau ab: Gegenüber 2001 hat die Zahl der Ausgänge nun etwas geringer um $56,7 \%$, die der Urlaube um $65,6 \%$ und die der Freigänger um 59,6\% abgenommen. Dieser gegenüber 2006 moderate Anstieg der Lockerungen fällt relativ betrachtet noch etwas größer aus, weil er sich auf eine zunehmend geringere Gefangenenzahl verteilt. Dennoch: Es ist in Hamburg üblich geworden, viele Gefangene nahezu unvorbereitet vor die Anstalt zu stellen. Die schwachen Kräfte der Einrichtungen der Entlassenhilfe müssen sehen, wie sie damit fertig werden. Sie stehen in der Gefahr, als Feigenblatt eines sich selbst genügenden Verwahrvollzuges missbraucht zu werden. Dabei ist es bei der anhaltend niedrigen und zunehmend niedrigeren Belegung zusätzlich unbegreiflich, dass die Mitarbeiterschaft nicht angehalten wird, die Zeit der Verbüßung deutlich offensiver zu einer optimalen Eingliederung der Gefangenen zu nutzen.

Was sagt die Justizbehörde dazu? Sie schmückt sich mit den Zahlen und verweist auf die Verringerung des Missbrauchs (Drs.18/4826). So ist die ohnehin geringe Versagerquote bei den Urlaubs- und Ausgangsgewährungen von 2001 auf 2007 von $0,55 \%$ auf $0,12 \%$ gesunken. Wer aber nichts riskiert, bewegt auch nichts. Wer glaubt, man könne im Trockenen Schwimmen und allein durch theoretische Unterweisungen Auto fahren lernen, riskiert Opfer. Unverantwortlich handelt, wer sich ressortegoistisch abkapselt und als nicht zuständig für vermeidbares Unglück erklärt.

Inzwischen hat die den Senator Lüdemann tragende Bürgerschaftsmehrheit diese Praxis, die Geist und Buchstaben des StVollzG von 1977 widerspricht, durch ein eigenes Landesgesetz festgeschrieben. Das fachliche Niveau gab der Senator in Interviews vor: „...Strafvollzug und Urlaub passen nicht zusammen. Ich kann mir nicht auf der einen Seite Gedanken machen über sechs Meter hohe Mauern und Außensicherung, so dass keiner...entweichen kann, und gleichzeitig sagen: ,21 Tage im Jahr hast Du Urlaub und kannst raus"“ (Die Welt, 31. 6. 06, s. auch Die Welt, 16. 1. 07; Hinz\&Kunzt, Jan. 2007). Man staune: Eine ansehnliche 
Population von Straftätern geringer bis mittlerer Schwere wird hoch übersichert verwahrt und der dies will und angeordnet hat, zeigt auf die Mauern etc. und schreit: Seht wie schlimm die sind! Das ist doppelt verwerflich, weil Ursache und Folge derselben Quelle eines bedenklichen Menschenbildes entspringen. Die Betroffenen sehen sich in einer Falle. Da mag der falsche Ausweg nahe liegen, die Fremddefinition der eigenen beizugeben und sich noch mehr in der Rolle des Bösen einzurichten...

\section{Es war einmal: Sozialtherapie und Übergangsvollzug}

Mit der Schließung/Verlagerung der drei kleinen, langjährig außerordentlich erfolgreichen und bundesweit vorbildlichen Übergangs- und Sozialtherapieanstalten erreichte die radikal restaurative „Vollzugspolitik“ des Gespanns Kusch/Lüdemann einen schmachvollen Höhepunkt. Im einzelnen:

Im Februar 2005 wurde die Übergangsanstalt Moritz-Liepmann-Haus (MLH) ersatzlos geschlossen. Sie wurde 1971 in zentraler Lage in HH-Altona eröffnet und verfügte über 38 Plätze für Männer und 7 für Frauen. Ihr Auftrag war es, langjährig einsitzende Gefangene in den letzten ca. neun Monaten ihrer Verbüßung auf dem Weg in die Freiheit zu begleiten. Konzeptionell beruhte dies auf einem stringenten sozialpädagogischen Konzept, das Selbsttätigkeit des Gefangenen (besonders bei der Arbeitssuche) mit intensiver Hilfe und Kontrolle durch Mitarbeiter/innen verband.

Im Dezember 2005 wurde die Sozialtherapeutische Anstalt $\mathrm{HH}$-Altengamme nach 21jähriger Tätigkeit geschlossen. Sie verfügte über 54 Plätze für Männer und 6 für Frauen. Die traditionsreiche, seit 1969 bestehende Sozialtherapie Bergedorf mit ihren 42 Haftplätzen wurde Außenstelle der JVA Fuhlsbüttel. Ihre ganz eigene Existenz wurde zerstört; viele Mitarbeiter/innen mussten in andere Anstalten wechseln.

In Bergedorf befanden sich schwerpunktmäßig eher neurotisch schwer gestörte Sexualstraftäter, aber auch Gewalttäter anderer Art, in Altengamme eher jüngere, dissozial ausagierende Personen mit oft gravierenden psychischen Strukturdefiziten und gefangene Frauen mit z.T. schwerer Delinquenz. Die Praxis beider Anstalten war strikt an den empirisch ermittelten Wirkfaktoren der integrativen Sozialtherapie orientiert: Dies umfasste vor allem intensiv strukturierte Therapie-, Trainings- und Wohngruppenkonzepte, ein hohes fachliches Niveau der Mitarbeiterschaft, ein behandlungsförderliches therapeutisches Milieu, die Einbeziehung der Bezugspersonen der Gefangenen und eine in jedem Einzelfall aufwendig begleitete Eingliederung in das Leben in Freiheit.

Mit der Schließung/Verlagerung der Sozialtherapie in die hoch gesicherte Großanstalt Fuhlsbüttel (ca. 1000 Haftplätze, davon ca. 100 Sozialtherapie) ging, wie vorauszusehen war, ihre spezifische Fachlichkeit weitgehend verloren. Es dominieren nun die baulichen, administrativen und sichernden Strukturen der Großanstalt, die davon geprägten Einstellungen traditioneller Art und die therapiebehindernden Strukturen der Gefangenensubkultur. Für den Therapieerfolg wesentliche Handlungsgrundlagen und Umgangsformen wurden knasttypisch zurechtgestutzt. Beiläufig wurde auch das in Altengamme vorhandene Therapieangebot für straffällige Frauen und die Vorteile eines behutsam koedukativen Strafvollzuges, den es sonst bundesweit nicht gibt, abgeschafft. Was bleibt, verdient, ohne grundlegende Änderungen, kaum noch den Namen „Sozialtherapie“...

Die drei Anstalten waren für Gefangene (und mittelbar für die mit ihnen arbeitenden Mitarbeiter/innen) eine erstrebenswerte Perspektive, ein solider Weg in eine bessere Zukunft. Jahr für Jahr wurden viele hoch vorbelastete Gefangene intensiv beraten und therapiert. Kaum einer wurde ohne Arbeits- oder Ausbildungsplatz und einen auch sonst geordneten sozialen Empfangsraum entlassen. So wurde in vielen Fällen ein sonst wahrscheinlicher Rückfall verhindert und weiteres Unglück von potentiellen Opfern, den Familien der Gefangenen und von ihnen selbst abgewendet. Der Erfolg dieser Arbeit ist wissenschaftlich belegt. Es bleibt unbegreiflich, warum dem Führungsduo in der Justizbehörde gestattet wurde, diese hoch komplexen und anspruchsvollen, dabei aber, auf alles gesehen, kostengünstigen Einrichtungen zu schließen, zu verlegen, zu vernichten

\section{Drei Schlussbemerkungen}

Auch früher war nicht alles in Ordnung. Zwar war auf dem Weg zu einem humanen, sowohl sicheren als auch eingliederungstauglichen Vollzug viel erreicht worden; jedoch gab es in Teilen noch erhebliche strukturelle, konzeptionelle und bauliche Mängel. Darüber berichten mehrere Senats- und Bürgerschaftsdrucksachen sowie Kommissionsberichte aus jener Zeit, die zugleich auch Programme für die Zukunft enthielten. Der Resozialisierungsauftrag blieb dabei unbestritten.

Auch in der Vergangenheit führten neue politische Konstellationen zu Kursänderungen. Neu und unerwartet war aber der durch die CDU durchgesetzte Traditionsbruch. Ausgehend von einer scharfen Wendung gegen den Resozialisierungsgedanken (hier zählen Taten, nicht Worte) beschädigten Kusch/Lüdemann gerade die Teile und Arbeitsformen des Hamburger Vollzuges nachhaltig, die sich bewährt hatten (z.B. Übergangsvollzug und Sozialtherapie in überschaubaren Einrichtungen) oder verbesserungsbedürftig, aber auch verbesserungswürdig waren (wie z.B. offener Vollzug und Lockerungspraxis). Diese Politik steht gegen rationale Vollzugsgrundsätze, gegen in der Mitarbeiterschaft personifizierte Erfahrungen und widerspricht wissenschaftlichen Erkenntnissen. Sie zerstört Brücken zwischen Straftätern und Gesellschaft, vergeudet Verbüßungszeit und schafft nicht mehr, sondern weniger Sicherheit.

Aber in den Ruinen des Hamburger Strafvollzuges gibt es noch Leben in funktionsfähigen Bereichen und im Umfeld der vielen engagierten Mitarbeiter/innen. Auch die Belegungssituation lädt zur Entkrampfung ein. So könnte z.B. Billwerder teilweise zur selbständigen offenen Anstalt zurückverwandelt werden; Glasmoor wäre umzubauen oder aufzugeben. Das MLH und Altengamme könnten in die alten Funktionen eingesetzt werden. Wenn letzteres verworfen wird, dann wäre das ganze Haus IV in der JVA Fuhlsbüttel zur selbstständigen Sozialtherapie umzurüsten und mit einer fachlich hoch qualifizierten Leitung, eigenem Budget, Mitarbeiterstamm und konzeptionellem Entfaltungspotential sowie einer eigenen Pforte zu versehen. Noch weisen die Zeichen aber in eine andere Richtung: Braucht das kleine Hamburg wirklich auch künftig ein eigenes und eher schäbiges Strafvollzugsgesetz? Und geht es wirklich um Chancen im nun propagierten „Chancenvollzug“, wenn seine Instrumente im tatsächlich gegebenen, vernachlässigenden Verwahrvollzug eher dazu dienen, die aus Perspektivlosigkeit resultierende Aggressivität der Gefangenen zu disziplinieren und so in Schach zu halten?

Aktualisierte Fassung eines Vortragstextes vom 09. 02. 2007, der in einer Broschüre des Forums Hamburger Straffälligenhilfe veröffentlicht wurde siehe auch www.forum-straffälligenhilfe-hh.de 